\title{
Raman spectrum and charge fluctuations in the copper-oxide superconductors
}

\author{
H. Nikšića ${ }^{a}$ E. Tutiš ${ }^{b}$ and S. Barišića

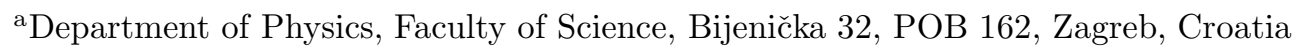 \\ ${ }^{\mathrm{b}}$ Institute of Physics, Bijenička 46, POB 304, Zagreb, Croatia
}

\begin{abstract}
The effect of the charge fluctuations on the electronic spectrum and the Raman spectrum of high temperature superconductors is examined within the slave boson approach. Instead of using the saddle point approximation for slave bosons, we confine ourselves to the non-crossing approximation (NCA) in summing the diagrams for the Green functions, thus obtaining the renormalized hole spectrum and its lifetime on equal footing. The electronic Raman spectrum is calculated, showing the characteristic featureless behaviour up to the frequency of the order of renormalized $\Delta_{p d}$ parameter. The dependence on the polarization of the incident and the scattered light agrees with experiments.
\end{abstract}

\section{Introduction}

One of the interesting properties of the HTSC copper-oxide materials is the featureless Raman spectrum for frequencies up to $1 \mathrm{eV}$, with characteristic polarization dependences [1, 2]. Here we reproduce qualitatively such behaviour within the slave boson formalism [3] for the $p$ - $d$ model with large $U_{d}$. In treating the slave bosons, we choose non-crossing $(N C A)$ diagrams for Green functions, following Kroha et al. [ $\mid$ a approach to the Anderson model. The resulting spectrum for the electron Green functions has three bands, "p"-band, "d"-band and the "in-gap"-band which contains the Fermi-level, consistently with some previous theoretical results and experimental observations.

\section{Model and method}

Within the $p$ - $d$ model for electrons in $\mathrm{CuO}_{2}$ we retain [5] the Coulomb repulsion $U_{d}$, the parameters $\epsilon_{d}, \epsilon_{p}$ and $t_{0}$, denoting respectively the energies of the hole in $\mathrm{Cu} 3 d_{x^{2}-y^{2}}$ and $O 2 p(\sigma)$ orbitals and the copper-oxygen hybridization. Taking the $U_{d} \rightarrow \infty$ limit, the slave boson substitution [3] $d_{\vec{n} \sigma} \rightarrow b_{\vec{n}}^{\dagger} f_{\vec{n} \sigma}$ is accompanied by the appearance of an additional, static, $\lambda$-field

$\left(H \rightarrow H+\sum_{\vec{n}} \lambda_{\vec{n}} \hat{Q}_{\vec{n}}\right)$ providing for the physical constraint $\hat{Q}_{\vec{n}} \equiv \sum_{\sigma} f_{\vec{n} \sigma}^{\dagger} f_{\vec{n} \sigma}+$ $b_{\vec{n}}^{\dagger} b_{\vec{n}}=1$. Following Kroha et al. [4], we neglect the fluctuations of $\lambda_{\vec{n}}$ around the mean field value $\Lambda$. This means that the states with $Q_{\vec{n}}$ different from unity became allowed. However, with $\lambda_{\vec{n}}$ fixed to $\Lambda$, the Hamiltonian, $H=H_{0}+H_{h y b}$ (with $H_{h y b}$ describing the hybridization of the copper orbital with the bonding combination of the oxygen orbitals),

$$
H_{0}=\sum_{k, \sigma}\left\{\epsilon_{p} p_{k, \sigma}^{\dagger} p_{k, \sigma}+\left(\epsilon_{d}+\Lambda\right) f_{k, \sigma}^{\dagger} f_{k, \sigma}\right\}+\sum_{k} \Lambda b_{k}^{\dagger} b_{k}
$$




$$
\begin{aligned}
& H_{h y b}=\sum_{k, \sigma}\left[t(k) p_{k, \sigma}^{\dagger} \sum_{q} f_{k+q, \sigma} b_{q}^{\dagger}+h . c .\right] \\
& t(k) \equiv 2 t_{0} \sqrt{\sin \left(k_{x} / 2\right)^{2}+\sin \left(k_{y} / 2\right)^{2}}
\end{aligned}
$$

conserves $\hat{Q}_{\vec{n}}$, so the states with different $Q_{\vec{n}}$ are not dynamically mixed. Therefore, we expect that on fixing $\left\langle\hat{Q}_{\vec{n}}\right\rangle=1$ our calculation will predominantly reflect the dynamics of the $Q_{\vec{n}}=1$ system. Further, following Kroha et al. we confine ourselves to $\mathrm{NCA}$ diagrams, thus discarding the $\mathrm{Cu}$ - $\mathrm{Cu}$ spin flip processes. Within the $N C A$ we get the set of Dyson equations (Fig. 1), which we solve nu-

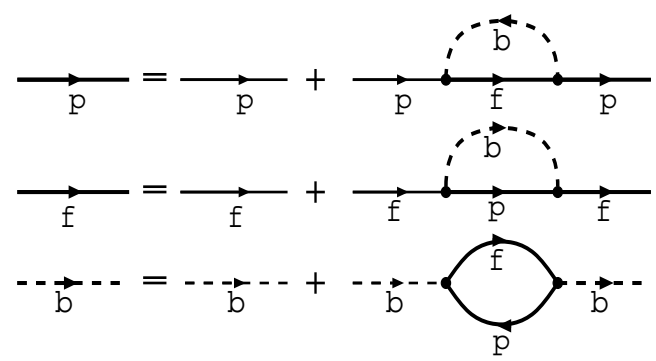

Figure 1: NCA diagrams for the Green functions.

merically. In absence of the

$b$-condensate, the one-particle Green functions for $b$ and $f$ fields are site-diagonal to all orders in the perturbation in Eq. (2). The Green function for the hole on the copper-site is obtained from the bare Green function $G_{p}^{0}$ on the oxygen-site and its self-energy corrections $\Sigma_{p}$ from Fig. 1.

$$
G_{d}(\omega, k)^{-1}=\Sigma_{p}(\omega, k)^{-1}-|t(k)|^{2} G_{p}^{0}(\omega, k) .
$$

\section{Hole spectrum and density of states}

The density of states (D.O.S.) for the copper and the oxygen site which we obtain for $1+\delta=1.14$ holes per unit cell are shown in Fig.2. We choose parameters $\Delta_{p d}=\epsilon_{p}-\epsilon_{d}=4.0 t_{0}$ $\left(t_{0} \approx 1 \mathrm{eV}\right), T=0.0135 t_{0}$ (temperature) and set $\epsilon_{p}=0$ for convenience. The values for $\Lambda$ and $\mu$ (Fermi-level) are $\Lambda=3.75 t_{0}$ and $\mu=-1.138 t_{0}$. For obvious physical reasons the D.O.S. accumulates around energies $\epsilon_{p}$ ("p"band), and $\epsilon_{d}$

( " $d$ "-band) of the unhybridized oxygen and copper orbitals. The Fermi level lies in a separate, resonant "in-gap"-band, similar to the result of the saddle point calculations in $b$ [5]. Note, however, that the band edges are smoothened out, due to the "disorder" produced by the slave boson.

\section{Raman spectrum}

The coupling of the electromagnetic field to electrons is introduced by the substitution,

$$
t c_{r}^{\dagger} c_{s} \rightarrow t c_{r}^{\dagger} c_{s} e^{\frac{i e}{\hbar c} \int_{s}^{r} \mathbf{A} \cdot \mathbf{d l}} .
$$




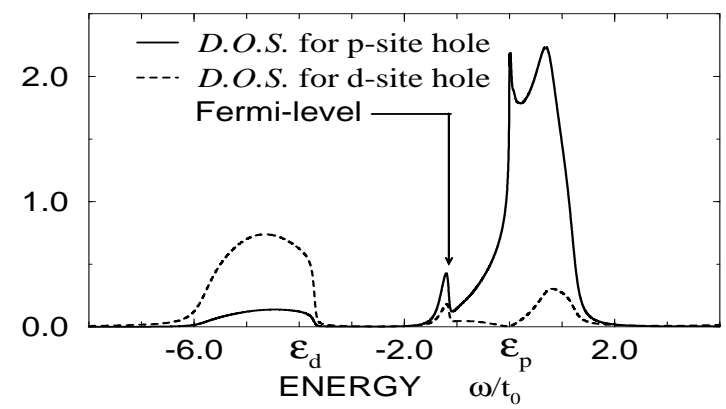

Figure 2: D.O.S. for $p$-site and $d$-site holes.

Expanding up to the quadratic terms in vector potential, we get usual $\vec{p} \cdot \vec{A}$ and $n \overrightarrow{A^{2}}$ electron-light coupling terms. Using these we calculate the resonant and nonresonant contributions to the transition matrix for the Raman scattering. The overall results are shown in Fig. 3. The featureless Raman spectrum,

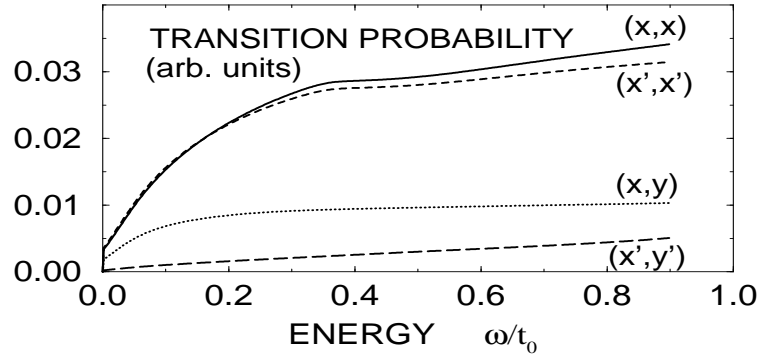

Figure 3: Transition probability for the Raman scattering for various polarizations.

in general agreement with experiment, extends up to the frequency of $1 \mathrm{eV}$ approximately. In our calculations this scale corresponds to the separation of the "in-gap"-band and "p"-band (corresponding to renormalized $\Delta_{p d}$ in the saddle point approximation [5]). Above that frequency (not probed experimentally) a steep increase of the Raman intensity occurs in our calculations due to the interband processes. The intensity of the spectrum significantly changes as a function of the polarization of the incident and the scattered light. This is due to the polarization dependence of the electron-light interaction, characteristic for the nearest neighbour hoping Hamiltonian in Eq. (2) (for example, the scattering via the $n \vec{A}^{2}$ coupling does not contribute at all for the ( $\left.\mathrm{x}^{\prime}, \mathrm{y}^{\prime}\right)$ polarization). The polarization dependence in our calculations (shown above) is in a qualitative accordinance with experiments [1, 2]. 


\section{References}

[1] T. Staufer, R. Hackl and P. Muller, Solid State Commun. 79 (1991) 409

[2] S. L. Cooper, D. Reznik, A. Kotz, M. A. Karlow, R. Liu, V. Klein, W. C. Lee, D. M. Ginsberg, B. W. Veal and A. P. Pauliskas, Phys. Rev. B 47 (1993) 8233;

[3] N. Read and D. M. Newns, J. Phys. C 16 (1983) L1055; 16 (1983) 3273

[4] J. Kroha, P. J. Hirschfeld, K. A. Muttalib, P. Wolfle, Solid State Commun. 83 (1992) 1003

[5] G. Kotliar, P. A. Lee, N. Read, Physica C 153-155 (1988) 538 